\title{
RUSH-LARSEN TIME-STEPPING METHODS OF HIGH ORDER FOR STIFF PROBLEMS IN CARDIAC ELECTROPHYSIOLOGY*
}

\author{
YVES COUDIÈRE ${ }^{\dagger}, \mathrm{CHARLIE} \mathrm{DOUANLA} \mathrm{LONTSI}^{\dagger}$, AND CHARLES PIERRE ${ }^{\ddagger}$
}

\begin{abstract}
The stability and accuracy of numerical methods for reaction-diffusion equations still need improvements, which prompts the development of high-order and stable time-stepping methods. This is particularly true in the context of cardiac electrophysiology, where reaction-diffusion equations are coupled with stiff systems of ordinary differential equations. So as to address these issues, much research on implicit-explicit methods and exponential integrators has been carried out during the past 15 years. In 2009, Perego and Veneziani [Electron. Trans. Numer. Anal., 35 (2009), pp. 234-256] proposed an innovative time-stepping scheme of order 2. In this paper we present an extension of this scheme to the orders 3 and 4, which we call Rush-Larsen schemes of order $k$. These new schemes are explicit multistep methods, which belong to the classical class of exponential integrators. Their general formulation is simple and easy to implement. We prove that they are stable under perturbation and convergent of order $k$. We analyze their Dahlquist stability and show that they have a very large stability domain provided that the stabilizer associated with the method captures well enough the stiff modes of the problem. We study their application to a system of equations that models the action potential in cardiac electrophysiology.
\end{abstract}

Key words. stiff equations, explicit high-order multistep methods, exponential integrators, stability and convergence, Dahlquist stability

AMS subject classifications. 65L04, 65L06, 65L20, 65L99

1. Introduction. This article concerns the problem of time integration of stiff reactiondiffusion equations, in particular when they are coupled to a system of ordinary differential equations (ODE). As developed below, for such problems the matters of stability and accuracy are of first importance. As a systemic example of these issues, we will consider the monodomain model in cardiac electrophysiology $[3,4,5]$. Given the heart domain $\Omega$ and the time interval $[0, T]$, it has the general form

$$
\frac{\partial v}{\partial t}=A v+f_{1}(v, \zeta)+s(x, t), \quad \frac{\mathrm{d} \zeta}{\mathrm{d} t}=f_{2}(v, \zeta),
$$

where $A$ is a diffusion operator. The unknown function $v: \Omega \times[0, T] \rightarrow \mathbb{R}$ is the transmembrane potential. The unknown function $\zeta: \Omega \times[0, T] \rightarrow \mathbb{R}^{p+q}$ gathers $p+q$ variables describing the state of the cell membrane. It incorporates $p$ gating variables and $q$ ionic concentrations. The source term $s(x, t)$ is an applied stimulation current. The reaction terms $f_{1}$ and $f_{2}$ model ionic currents across the cell membrane and are called ionic models. Ionic models have originally been developed by Hodgkin and Huxley [19] in 1952. Highly detailed ionic models specific to cardiac cells have been designed since the 1960's, such as the Beeler and Reuter (BR) model [1] or the ten Tusscher, Noble, Noble, and Panfilov (TNNP) model [29]. A comprehensive review is available in [28].

There are two major difficulties for numerical simulations in cardiac electrophysiology. First, the nonlinear functions $f_{1}$ and $f_{2}$ in equation (1.1) induce expensive computations of the mappings $(v, \zeta) \rightarrow f_{i}(v, \zeta)$. For example, the TNNP model [29] involves the computation of 50 scalar exponentials, which has to be performed for each mesh node to approximate

\footnotetext{
*Received October 6, 2017. Accepted June 14, 2020. Published online on June 29, 2020. Recommended by A. Ostermann.

†Université de Bordeaux, IMB CNRS UMR 5251, France. Carmen Research Team, Inria. IHU Liryc, Electrophysiology and Heart Modeling Institute, fondation Bordeaux Université

${ }^{\ddagger}$ Laboratoire de Mathématiques et de leurs Applications, CNRS UMR 5142, Université de Pau et des Pays de l'Adour, France (charles.pierre@univ-pau.fr).
} 
the solutions of the partial differential equation (1.1). They represent the predominant computational load during numerical simulations, and their total computational amount needs to be maintained as low as possible. Fully implicit time-stepping methods, which require a nonlinear solver, are therefore avoided. Second, the equations (1.1) are stiff, but since implicit methods are not affordable, numerical instabilities are challenging to manage. More precisely, the stiffness is caused by the presence of different space and time scales. The solutions of equation (1.1) display sharp wavefronts. Typically, the scaling factor between the fast and slow variables ranges from 100 to 1000 . This is commonly coped with by resorting to very fine space and time discretization grids, associated with high computational costs.

In this context, our strategy for solving problem (1.1) is to use high-order methods, so as to have accurate simulations with coarser space and time discretization grids. A high-order time-stepping method that fulfills the two following conditions is required: it must have strong stability properties and has to be explicit for the reaction terms. To this aim, we will focus on the time integration of stiff ODE systems of the form

$$
\frac{\mathrm{d} y}{\mathrm{~d} t}=f(t, y), \quad y(0)=y_{0},
$$

in which the nonlinear function $f:\left[0,+\infty\left[\times \mathbb{R}^{N} \rightarrow \mathbb{R}^{N}\right.\right.$ (e.g., $N=p+q$ for the ionic models presented above) may be split as $f(t, y)=a(t, y) y+b(t, y)$. This leads to a formulation more suited to our needs,

$$
\frac{\mathrm{d} y}{\mathrm{~d} t}=a(t, y) y+b(t, y), \quad y(0)=y_{0} .
$$

It involves the nonlinear term $b(t, y)$ and the operator $y \in \mathbb{R}^{N} \mapsto a(t, y) y \in \mathbb{R}^{N}$, which can be easily linearized as, e.g., $a\left(t, y_{n}\right) y$. This function $a(t, y)$ will be inserted into the numerical scheme in order to stabilize the computations. It will be called the stabilizer in the sequel. In practice, the function $a(t, y)$ may be related to the Jacobian of the system $\partial_{y} f(t, y)$. However, no a priori definition of the stabilizer is made (such as $a(t, y)=\partial_{y} f(t, y)$ ) because we plan to analyze the formulation in (1.3) in general. This will allow us, for instance, to define the stabilizer as an approximation of the Jacobian, for technical reasons detailed below. This approach is relevant in cardiac electrophysiology, where the fastest variables are gating variables that are given by the first $p$ equations of the ODE system $\mathrm{d} \zeta / \mathrm{d} t=f_{2}(v, \zeta)$ in (1.1). They have the general form

$$
\frac{\mathrm{d} \zeta_{i}}{\mathrm{~d} t}=\frac{\zeta_{i, \infty}(v)-\zeta_{i}}{\tau_{i}(v)}
$$

(see Section 5.1), which motivates the reformulation (1.3) with the diagonal stabilizer $a=\operatorname{diag}\left(-1 / \tau_{i}\right)$.

Exponential integrators are well suited in this framework; we refer to [12, 16, 23] for general reviews. They have been widely studied for the semilinear equation $\partial_{t} y=A y+b(t, y)$; see, e.g., $[7,14,15,18,21,30]$. Exponential integrators commonly define a time iteration based on the exact solution of an equation of the form $\partial_{t} y=A y+p(t)$, where $p(t)$ is a polynomial. It is usually defined with the functions $\left(\varphi_{k}\right)_{k \geq 0}$,

$$
\varphi_{0}(z)=\mathrm{e}^{z}, \quad \varphi_{j+1}(z)=\frac{\varphi_{j}(z)-1 / j !}{z},
$$

introduced by Nørsett [24]. In general, it requires to compute a matrix exponential applied to a vector, like $\mathrm{e}^{t A} y$. This is the supplementary cost associated with exponential integrators. A gain in stability is expected when $A$ is the predominant stiff part of the equation. 
The target equation (1.3) incorporates a non-constant linear part $a(t, y)$, and exponential integrators have been less studied in that case. Exponential integrators of Adams type for a non-constant linear part have been first considered by Lee and Preiser [20] in 1978 and by Chu [2] in 1983. Recently, Ostermann et al. [17] developed and analyzed the linearized exponential Adams method. In general, the original equation (1.2) is formulated after each time step as $\frac{\mathrm{d} y}{\mathrm{~d} t}=J_{n} y+c_{n}(t, y)$ involving the Jacobian matrix $J_{n}=\partial_{y} f\left(t_{n}, y_{n}\right)$ and the correction function $c_{n}(t, y)=f(t, y)-J_{n} y$. This has several drawbacks. It requires the computation of a matrix exponential applied to a vector with a different matrix at each time step. Moreover, stabilization can be performed on the fast variables only in case that they are known in advance, e.g., because of modeling assumptions or of our physical understanding of the problem. In this case, using the full Jacobian as the stabilizer will cause unnecessary computational efforts.

As an alternative, the stabilizer can be set to a part or an approximation of the Jacobian. This had already been proposed by Nørsett [24] in 1969 and has been analyzed in [31], [26], and [6] for exponential Rosenbrock, exponential Runge-Kutta, and exponential Adams methods, respectively. For exponential Adams methods, equation (1.3) is reformulated after each time step as

$$
\frac{\mathrm{d} y}{\mathrm{~d} t}=a_{n} y+c_{n}(t, y), \quad a_{n}=a\left(t_{n}, y_{n}\right), \quad c_{n}(t, y)=f(t, y)-a_{n} y .
$$

The resulting scheme with a time step $h>0$ is (see the details in $[6,17]$ )

$$
y_{n+1}=y_{n}+h\left(\varphi_{1}\left(a_{n} h\right)\left(a_{n} y_{n}+\gamma_{1}\right)+\varphi_{2}\left(a_{n} h\right) \gamma_{2}+\ldots \varphi_{k}\left(a_{n} h\right) \gamma_{k}\right),
$$

where the numbers $\gamma_{i}$ are the coefficients of the Lagrange interpolation polynomial of $c_{n}(t, y)$ (in a classical $k$-step setting) and the functions $\varphi_{j}$ are given by (1.4).

Independently, Perego and Veneziani [25] presented in 2009 an innovative exponential integrator of order 2 of a different nature. They proposed a scheme of the form

$$
y_{n+1}=y_{n}+h \varphi_{1}\left(\alpha_{n} h\right)\left(\alpha_{n} y_{n}+\beta_{n}\right)
$$

involving two coefficients $\alpha_{n}$ and $\beta_{n}$ to be computed at each time step. The resulting scheme has a very simple definition and is, in particular, simpler than the exponential Adams integrators (1.5). The essential difference with the previous approaches is that $\alpha_{n} \neq a\left(t_{n}, y_{n}\right)$ but instead is fixed for the scheme to be consistent of order 2. Specifically, the coefficients $\alpha_{n}$ and $\beta_{n}$ are given by

$$
\alpha_{n}=\frac{3}{2} a_{n}-\frac{1}{2} a_{n-1}, \quad \beta_{n}=\frac{3}{2} b_{n}-\frac{1}{2} b_{n-1} \quad \text { with } \quad a_{j}=a\left(t_{j}, y_{j}\right) \quad b_{j}=b\left(t_{j}, y_{j}\right) .
$$

Perego and Veneziani presented their scheme as a "generalization of the Rush-Larsen method" in reference to the Rush-Larsen scheme [27] commonly used in electrophysiology.

This scheme resembles the Magnus integrator introduced by Hochbruck et al. in [13] for the time-dependent Schrödinger equation $i y^{\prime}=H(t) y$ and extended by Gonzàlez et al. in [9] to parabolic equations with time-dependent linear part $y^{\prime}=a(t) y+b(t)$. The second-order Magnus integrator also formulates as (1.6) but with $\alpha_{n}=a\left(t_{n+1 / 2}\right)$ and $b_{n}=b\left(t_{n+1 / 2}\right)$. The scheme of Perego and Veneziani generalizes the second-order Magnus integrator to the case where $a=a(t, y)$ and $b=b(t, y)$ : it presents an approximation of the unknown terms $a\left(t_{n+1 / 2}, y\left(t_{n+1 / 2}\right)\right)$ and $b\left(t_{n+1 / 2}, y\left(t_{n+1 / 2}\right)\right)$ using a two-point interpolation.

In this paper we will study schemes in the form of (1.6). We will show that they also exist at the orders 3 and 4 and will exhibit explicit definitions of the two coefficients $\alpha_{n}$ and 
$\beta_{n}$. The schemes will be referred to as Rush-Larsen schemes of order $k$ (shortly denoted by $\mathrm{RL}_{k}$ ) in the continuation of the denomination used in [25]. They will be shown to be stable under perturbation and convergent of order $k$. We also present the Dahlquist stability analysis for the $\mathrm{RL}_{k}$ schemes. It is a practical tool that allows one to scale the time step $h$ with respect to the variations of the function $f(t, y)$ in problem (1.2); see, e.g., [11]. The splitting $f(t, y)=a(t, y) y+b(t, y)$ may be arbitrary, but obviously the choice of the stabilizer term $a(t, y)$ is critical for the stability of the method. When considering time-dependent stabilizers, the stability domain depends on this splitting. We compute stability domains numerically and show that they are much larger if $a(t, y)$ captures the variations of $f(t, y)$ than in absence of stabilization (i.e., when $a(t, y)=0$ ). We finally evaluate the performances of the $\mathrm{RL}_{k}$ methods as applied to the membrane equation in cardiac electrophysiology. They are compared to the exponential Adams integrators (1.5). The two methods have a very similar robustness with respect to stiffness, allowing stable computations with large time steps. For the considered test case, the $\mathrm{RL}_{3}$ and $\mathrm{RL}_{4}$ schemes are more accurate for large time steps.

The paper is organized as follows: The $\mathrm{RL}_{k}$ schemes are derived in Section 2, and their numerical analysis is performed in Sections 2 and 3. The Dahlquist stability analysis is completed in Section 4. The numerical results are presented in Section 5. The paper ends with a conclusion in Section 6 . In the following, $h$ denotes the time step and $t_{n}=n h$ are the associated time instants starting at $t_{0}=0$.

2. Definition of $\mathbf{R L}_{\boldsymbol{k}}$ schemes and consistency. Let us consider a solution $y(t)$ of equation (1.3) in a time interval $[0, T]$. It is recalled that the scheme (1.6) is consistent of order $k$ if, given a time step $h$, a time instant $k h \leq t_{n} \leq T-h$, and the numerical approximation $y_{n+1}$ in (1.6) computed with $y_{n-j}=y\left(t_{n-j}\right)$, for $j=0, \ldots, k-1$, we have

$$
\left|y_{n+1}-y\left(t_{n}+h\right)\right| \leq C h^{k+1},
$$

for a constant $C$ only depending on the data $a, b, y_{0}$, and $T$ of the problem (1.3).

LEMma 2.1. Assume that the functions $a(t, y)$ and $b(t, y)$ are $\mathcal{C}^{k}$ regular on $[0, T] \times \mathbb{R}^{N}$. Moreover, assume that $a(t, y)$ is diagonal $\left(a(t, y)=\operatorname{diag}\left(a_{i}(t, y)\right)\right)$ or constant. Then the scheme in (1.6) is consistent of order $k$, for $k=2,3,4$, if

- for $k=2$, we have

$$
\alpha_{n}=a_{n}+\frac{1}{2} a_{n}^{\prime} h+O\left(h^{2}\right), \quad \text { and } \quad \beta_{n}=b_{n}+\frac{1}{2} b_{n}^{\prime} h+O\left(h^{2}\right) ;
$$

- for $k=3$, we have

$$
\begin{aligned}
& \alpha_{n}=a_{n}+\frac{1}{2} a_{n}^{\prime} h+\frac{1}{6} a_{n}^{\prime \prime} h^{2}+O\left(h^{3}\right) \quad \text { and } \\
& \beta_{n}=b_{n}+\frac{1}{2} b_{n}^{\prime} h+\frac{1}{12}\left(a_{n}^{\prime} b_{n}-a_{n} b_{n}^{\prime}\right) h^{2}+O\left(h^{3}\right) ;
\end{aligned}
$$

- for $k=4$, we have

$$
\begin{aligned}
\alpha_{n}=a_{n} & +\frac{1}{2} a_{n}^{\prime} h+\frac{1}{6} a_{n}^{\prime \prime} h^{2}+\frac{1}{24} a_{n}^{\prime \prime \prime} h^{3}+O\left(h^{4}\right), \quad \text { and } \\
\beta_{n}=b_{n} & +\frac{1}{2} b_{n}^{\prime} h+\frac{1}{12}\left(a_{n}^{\prime} b_{n}-a_{n} b_{n}^{\prime}\right) h^{2} \\
& +\frac{1}{24}\left(b_{n}^{\prime \prime \prime}+a_{n}^{\prime \prime} b_{n}-a_{n} b_{n}^{\prime \prime}\right) h^{3}+O\left(h^{4}\right)
\end{aligned}
$$




\section{ETNA}

Kent State University and

Johann Radon Institute (RICAM)

where $a_{n}^{\prime}, a_{n}^{\prime \prime}, a_{n}^{\prime \prime \prime}$ and $b_{n}^{\prime}, b_{n}^{\prime \prime}, b_{n}^{\prime \prime \prime}$ denote the successive derivatives at time $t_{n}$ of the functions $t \mapsto a(t, y(t))$ and $t \mapsto b(t, y(t))$.

REMARK 2.2. The assumption " $a(t, y)$ is diagonal or constant" in Lemma 2.1 has the following origin: To analyze the consistency of the scheme, we will compute a Taylor expansion in $h$ of the scheme in (1.6). This expansion is derived from Taylor expansions of $\alpha_{n}$ and $\beta_{n}$. For the sake of simplicity, assume the simple form $\alpha_{n}=\alpha_{n, 0}+h \alpha_{n, 1}$. We need to expand $\varphi_{1}\left(\alpha_{n} h\right)$ as a power series in $h$, where the function $\varphi_{1}$ is analytic on $\mathbb{C}$. However, in the matrix case, the equality, $\varphi_{1}(M+N)=\varphi_{1}(M)+\varphi_{1}^{\prime}(M) N+\ldots+\varphi_{1}^{(i)}(M) N^{i} / i !+\ldots$ holds if $M$ and $N$ are commutative matrices. Therefore one cannot expand $\varphi_{1}\left(\alpha_{n} h\right)$ without the assumptions that $\alpha_{n, 0}$ and $\alpha_{n, 1}$ commute. This difficulty vanishes if $a(t, y)$ is constant or a varying diagonal matrix.

Proof. Consider equation (1.3) on the closed time interval $[0, T]$ and its solution, the function $y$. Since the functions $a$ and $b$ are $\mathcal{C}^{k}$ regular on $[0, T] \times \mathbb{R}^{N}$, the solution $y(t)$ is $\mathcal{C}^{k+1}$ regular on $[0, T]$. Its derivatives up to order $k+1$ are bounded by constants only depending on the data of problem (1.3) and on $T$. The Taylor expansion of $y$ at time instant $t_{n}$ is

$$
y\left(t_{n}+h\right)=y\left(t_{n}\right)+\sum_{j=1}^{k} \frac{s_{j}}{j !} h^{j}+O\left(h^{k+1}\right),
$$

with $s_{j}=y^{(j)}\left(t_{n}\right)$. Using that $y^{\prime}=a y+b$, we get that

$$
\begin{aligned}
s_{1}= & a_{n} y_{n}+b_{n}, \\
s_{2}= & \left(a_{n}^{\prime}+a_{n}^{2}\right) y_{n}+a_{n} b_{n}+b_{n}^{\prime}, \\
s_{3}= & \left(a_{n}^{\prime \prime}+3 a_{n} a_{n}^{\prime}+a_{n}^{3}\right) y_{n}+b_{n}^{\prime \prime}+a_{n} b_{n}^{\prime}+2 a_{n}^{\prime} b_{n}+a_{n}^{2} b_{n}, \\
s_{4}= & \left(a_{n}^{\prime \prime \prime}+4 a_{n}^{\prime \prime \prime} a_{n}+3 a_{n}^{\prime 2}+6 a_{n}^{\prime} a_{n}^{2}+a_{n}^{4}\right) y_{n} \\
& \quad+b_{n}^{\prime \prime \prime}+b_{n}^{\prime \prime} a_{n}+3 a_{n}^{\prime \prime} b_{n}+5 a_{n}^{\prime} a_{n} b_{n}+3 a_{n}^{\prime} b_{n}^{\prime}+a_{n}^{3} b_{n}+a_{n}^{2} b_{n}^{\prime} .
\end{aligned}
$$

Series expansions in $h$ for $\alpha_{n}$ and for $\beta_{n}$ are introduced as

$$
\begin{aligned}
& \alpha_{n}=\alpha_{n, 0}+\alpha_{n, 1} h+\cdots+\alpha_{n, k-1} h^{k-1}+O\left(h^{k}\right), \\
& \beta_{n}=\beta_{n, 0}+\beta_{n, 1} h+\cdots+\beta_{n, k-1} h^{k-1}+O\left(h^{k}\right) .
\end{aligned}
$$

If the matrix $a(t, y)$ is diagonal or constant (see Remark 2.2), then the Taylor expansion of the numerical solution $y_{n+1}$ in (1.6) can be performed:

$$
y_{n+1}=y\left(t_{n}\right)+\sum_{j=1}^{k} \frac{r_{j}}{j !} h^{j}+O\left(h^{k+1}\right) .
$$

A direct computation of the $r_{j}$ gives

$$
\begin{aligned}
& r_{1}=\alpha_{n, 0} y_{n}+\beta_{n, 0}, \\
& r_{2}=\left(2 \alpha_{n, 1}+\alpha_{n, 0}^{2}\right) y_{n}+2 \beta_{n, 1}+\alpha_{n, 0} \beta_{n, 0}, \\
& r_{3}=\left(6 \alpha_{n, 2}+\alpha_{n, 0}^{3}+6 \alpha_{n, 0} \alpha_{n, 1}\right) y_{n}+3 \alpha_{n, 1} \beta_{n, 0}+6 \beta_{n, 2}+\alpha_{n, 0}^{2} \beta_{n, 0}+3 \alpha_{n, 0} \beta_{n, 1}, \\
& r_{4}=\left(24 \alpha_{n, 0} \alpha_{n, 2}+24 \alpha_{n, 3}+12 \alpha_{n, 1} \alpha_{n, 0}^{2}+12 \alpha_{n, 1}^{2}+\alpha_{n, 0}^{4}\right) y_{n} \\
& \quad+12 \alpha_{n, 2} \beta_{n, 0}+24 \beta_{n, 3}+12 \alpha_{n, 0} \beta_{n, 2}+12 \alpha_{n, 1} \beta_{n, 1}+4 \alpha_{n, 0}^{2} \beta_{n, 1} \\
& \quad+8 \alpha_{n, 0} \alpha_{n, 1} \beta_{n, 0}+\alpha_{n, 0}^{3} \beta_{n, 0},
\end{aligned}
$$


where $y_{n}$ denotes $y\left(t_{n}\right)$. The condition to be consistent of order $k$ is: $r_{i}=s_{i}$, for $1 \leq i \leq k$. The consistency conditions in Lemma 2.1 are obtained by recursively solving these relations.

We then can state our main result, which includes the definition of the $\mathrm{RL}_{k}$ schemes.

THEOREM 2.3. Assume (as in Lemma 2.1) that the functions $a(t, y)$ and $b(t, y)$ are $\mathcal{C}^{k}$ regular on $[0, T] \times \mathbb{R}^{N}$ and that $a(t, y)$ is diagonal or constant. Then, the three schemes defined for $k=2,3,4$ by equation (1.6) and the following coefficients,

- for $k=2$,

$$
\alpha_{n}=\frac{3}{2} a_{n}-\frac{1}{2} a_{n-1}, \quad \beta_{n}=\frac{3}{2} b_{n}-\frac{1}{2} b_{n-1},
$$

- for $k=3$,

$$
\begin{aligned}
& \alpha_{n}=\frac{1}{12}\left(23 a_{n}-16 a_{n-1}+5 a_{n-2}\right), \\
& \beta_{n}=\frac{1}{12}\left(23 b_{n}-16 b_{n-1}+5 b_{n-2}\right)+\frac{h}{12}\left(a_{n} b_{n-1}-a_{n-1} b_{n}\right),
\end{aligned}
$$

- for $k=4$,

$$
\begin{aligned}
\alpha_{n}= & \frac{1}{24}\left(55 a_{n}-59 a_{n-1}+37 a_{n-2}-9 a_{n-3}\right) \\
\beta_{n}= & \frac{1}{24}\left(55 b_{n}-59 b_{n-1}+37 b_{n-2}-9 b_{n-3}\right) \\
& \quad+\frac{h}{12}\left(a_{n}\left(3 b_{n-1}-b_{n-2}\right)-\left(3 a_{n-1}-a_{n-2}\right) b_{n}\right)
\end{aligned}
$$

with $a_{j}=a\left(t_{j}, y_{j}\right)$ and $b_{j}=b\left(t_{j}, y_{j}\right)$, are consistent of order $k$.

The three methods stated above are called Rush-Larsen methods of order $k$ and are denoted by $R L_{k}$. They are explicit and $k$-step methods.

REMARK 2.4. If the matrix $a$ is a constant, $a(t, y)=A$, then we have $\alpha_{n}=A$ for all three methods. In this case, the expressions of the coefficients $\beta_{n}$ in Theorem 2.3 for $k=3,4$ simplify as follows:

$$
\begin{array}{rlrl}
\mathrm{RL}_{3} \text { case: } & \beta_{n}=\frac{1}{12}\left(23 b_{n}-16 b_{n-1}+5 b_{n-2}\right)-\frac{h}{12} A\left(b_{n}-b_{n-1}\right) ; \\
\text { RL } 4 \text { case: } \quad \beta_{n}=\frac{1}{24}\left(55 b_{n}-59 b_{n-1}+37 b_{n-2}-9 b_{n-3}\right) \\
-\frac{h}{12} A\left(2 b_{n}-3 b_{n-1}+b_{n-2}\right) .
\end{array}
$$

Proof. The result is a direct consequence of backwards differentiation formulas, which we recall first. The derivatives of a real function $f$ at the time instant $t_{n}$ can be approximated as follows (with common notations):

- first derivative,

$$
\begin{aligned}
f_{n}^{\prime} & =\frac{f_{n}-f_{n-1}}{h}+O(h) \\
& =\frac{1}{2 h}\left(3 f_{n}-4 f_{n-1}+f_{n-2}\right)+O\left(h^{2}\right) \\
& =\frac{1}{6 h}\left(11 f_{n}-18 f_{n-1}+9 f_{n-2}-2 f_{n-3}\right)+O\left(h^{3}\right)
\end{aligned}
$$


- second derivative,

$$
\begin{aligned}
f_{n}^{\prime \prime} & =\frac{1}{h^{2}}\left(f_{n}-2 f_{n-1}+f_{n-2}\right)+O(h) \\
& =\frac{1}{h^{2}}\left(2 f_{n}-5 f_{n-1}+4 f_{n-2}-f_{n-3}\right)+O\left(h^{2}\right) ;
\end{aligned}
$$

- third derivative,

$$
f_{n}^{\prime \prime \prime}=\frac{1}{h^{3}}\left(f_{n}-3 f_{n-1}+3 f_{n-2}-f_{n-3}\right)+O(h) .
$$

With these formulas, the consistency condition at order 3 for the coefficient $\alpha_{n}$ becomes

$$
\begin{aligned}
\alpha_{n} & =a_{n}+\frac{1}{2} a_{n}^{\prime} h+\frac{1}{6} a_{n}^{\prime \prime} h^{2}+O\left(h^{3}\right) \\
& =a_{n}+\frac{1}{4}\left(3 a_{n}-4 a_{n-1}+a_{n-2}\right)+\frac{1}{6}\left(a_{n}-2 a_{n-1}+a_{n-2}\right)+O\left(h^{3}\right) \\
& =\frac{1}{12}\left(23 a_{n}-16 a_{n-1}+5 a_{n-2}\right)+O\left(h^{3}\right) .
\end{aligned}
$$

We retrieve the definition of $\alpha_{n}$ for the $\mathrm{RL}_{3}$ scheme. The same proof holds for $\beta_{n}$ and extends to order 4 .

3. Stability under perturbation and convergence. We refer to [10, Ch. III-8] for the definitions of convergence and of stability under perturbation. For the analysis of time-stepping methods, it is commonly assumed that $f$ in equation (1.2) is uniformly Lipschitz with respect to its second variable $y$. This hypothesis will be replaced by assumptions based on the formulation (1.3). Precisely, it will be assumed that

$$
a(t, y) \text { is bounded, } \quad a(t, y), b(t, y) \text { are uniformly Lipschitz in } y \text {. }
$$

The Lipschitz constants of $a$ and $b$ are denoted by $L_{a}$ and $L_{b}$, respectively. The upper bound for $|a(t, y)|$ is denoted by $M_{a}$.

PROPOSITION 3.1. If assumption (3.1) holds, then the $R L_{k}$ schemes are stable under perturbation for $k=2,3$, 4. In addition, also for $k=2,3,4$, if the consistency assumptions of Theorem 2.3 are satisfied $\left(a(t, y)\right.$ and $b(t, y)$ are $\mathcal{C}^{k}$ regular and $a(t, y)$ is diagonal or constant), then the $R L_{k}$ scheme is convergent of order $k$.

Stability under perturbation together with consistency implies (nonstiff) convergence; see, e.g., [10] or [6], where the current setting has been detailed. Therefore the proof of the convergence statement in Proposition 3.1 is immediate and will not be recalled here.

The following definitions are necessary to prove Proposition 3.1: Equation (1.2) is considered on $E=\mathbb{R}^{N}$ with the max-norm $|\cdot|$. A final time $T>0$ is considered. The space of $N \times N$ matrices is equipped with the operator norm $\|\cdot\|$ associated with $|\cdot|$. The space $E^{k}$ is equipped with the max-norm $|Y|_{\infty}=\max _{1 \leq i \leq k}\left|y_{i}\right|$ with $Y=\left(y_{1}, \ldots, y_{k}\right)$. The $\mathrm{RL}_{k}$ scheme is defined by the mapping

$$
s_{t, h}: Y=\left(y_{1}, \ldots, y_{k}\right) \in E^{k} \longrightarrow s_{t, h}(Y) \in E
$$

with

$$
s_{t, h}(Y)=y_{k}+h \varphi_{1}\left(\alpha_{t, h}(Y) h\right)\left(\alpha_{t, h}(Y) y_{k}+\beta_{t, h}(Y)\right)
$$




\section{ETNA}

Kent State University and

Johann Radon Institute (RICAM)

in such a way that the scheme in (1.6) reads $y_{n+1}=s_{t_{n}, h}\left(y_{n-k+1}, \ldots, y_{n}\right)$. The functions $\alpha_{t, h}$ and $\beta_{t, h}$ map the vector $Y$ of the $k$ previous values to the values $\alpha_{n}$ and $\beta_{n}$ given in Theorem 2.3. For instance, the function $\alpha_{t, h}(Y)$ for $k=3$ (the $\mathrm{RL}_{3}$ scheme) reads

$$
\alpha_{t, h}(Y)=\frac{1}{12}\left(23 a\left(t, y_{3}\right)-16 a\left(t-h, y_{2}\right)+5 a\left(t-2 h, y_{1}\right)\right)
$$

A first technique to prove stability under perturbation consists in showing that the function $s_{t, h}$ is globally Lipschitz in $Y$. To this aim, the derivative $\partial_{Y} s_{t, h}$ has to be analyzed. As developed in Remark 2.2, this implies restrictions on the function $a(t, y)$ : it has to be either diagonal or constant. A second technique consists in proving the following two stability conditions:

$$
\begin{aligned}
\left|s_{t, h}(Y)-s_{t, h}(Z)\right| & \leq|Y-Z|_{\infty}\left(1+C h\left(|Y|_{\infty}+1\right)\right) \\
\left|s_{t, h}(Y)\right| & \leq|Y|_{\infty}(1+C h)+C h
\end{aligned}
$$

for all $Y$ and $Z$ in $E^{k}$, and where the constant $C$ depends only on the data $a, b, y_{0}$ in equation (1.3) and on the final time $T$. These are sufficient conditions for stability under perturbation as proved in [6, Section 2]. We will use the conditions (3.2) and (3.3) here because they are more general and give rise to less computations.

The core of the proof is the following property of the $\operatorname{RL}_{k}$ scheme. For $Y=\left(y_{1}, \ldots, y_{k}\right) \in$ $E^{k}$, we have

$$
s_{t, h}(Y)=z(t+h) \quad \text { for } z^{\prime}=\alpha_{t, h}(Y) z+\beta_{t, h}(Y), \quad z(t)=y_{k} .
$$

It will be used together with the following Gronwall inequality (see [8, Lemma 196, p.150]): Suppose that $z(t)$ is a $\mathcal{C}^{1}$ function and that there exists $M_{1}>0$ and $M_{2}>0$ such that $\left|z^{\prime}(t)\right| \leq M_{1}|z(t)|+M_{2}$ for all $t \in\left[t_{0}, t_{0}+h\right]$. Then

$$
\forall t \in\left[t_{0}, t_{0}+h\right], \quad|z(t)| \leq \mathrm{e}^{M_{1}\left(t-t_{0}\right)}\left(\left|z\left(t_{0}\right)\right|+M_{2}\left(t-t_{0}\right)\right) .
$$

Proof of Proposition 3.1. In this proof, we always assume that $0 \leq h, t \leq T$, and denote by $C_{i}$ a constant that depends only on the data $a, b$, and $T$ of problem (1.3). With the assumptions in (3.1) and the definitions of $\alpha_{n}(k=2,3,4)$ in Theorem 2.3, the function $\alpha_{t, h}$ is uniformly Lipschitz with a Lipschitz constant equal to $L_{\alpha}$. Moreover, we have the uniform bound $\left\|\alpha_{t, h}\right\| \leq M_{\alpha}$. Since the function $b(t, y)$ is uniformly Lipschitz with respect to $y$ and since $0 \leq t \leq T$, we have

$$
|b(t, y)| \leq|b(t, 0)|+|b(t, y)-b(t, 0)| \leq K_{b}(1+|y|)
$$

with $K_{b}=\max \left(L_{b}, \sup _{0 \leq t \leq T}|b(t, 0)|\right)$. For the $\mathrm{RL}_{3}$ scheme, we have

$$
\left|\beta_{t, h}(Y)\right|_{\infty} \leq \frac{11}{3} K_{b}\left(1+|Y|_{\infty}\right)+\frac{h}{12} M_{a} 2 K_{b}\left(1+|Y|_{\infty}\right) \leq C_{1}\left(1+|Y|_{\infty}\right)
$$

The same inequality holds for the $\mathrm{RL}_{2}$ and $\mathrm{RL}_{4}$ schemes. Afterwards, we can apply these bounds to the differential equation in (3.4),

$$
\left|z^{\prime}\right|=\left|\alpha_{t, h}(Y) z+\beta_{t, h}(Y)\right| \leq M_{\alpha}|z|+C_{1}\left(1+|Y|_{\infty}\right) .
$$

The initial state is $|z(t)|=\left|y_{k}\right| \leq|Y|_{\infty}$. Finally, the Gronwall inequality (3.5) yields, for $t \leq \tau \leq t+h$,

$$
\begin{aligned}
|z(\tau)| & \leq \mathrm{e}^{M_{\alpha} h}\left(|Y|_{\infty}+h C_{1}\left(1+|Y|_{\infty}\right)\right) \\
& \leq \mathrm{e}^{M_{\alpha} h}\left(|Y|_{\infty}\left(1+C_{1} h\right)+C_{1} h\right) \\
& \leq|Y|_{\infty}\left(1+C_{2} h\right)+C_{2} h
\end{aligned}
$$


by bounding the exponential with an affine function for $0 \leq h \leq T$. This gives the stability condition (3.3) for $\tau=t+h$.

For the $\mathrm{RL}_{2}$ scheme, the function $\beta_{t, h}$ is uniformly Lipschitz. For the $\mathrm{RL}_{3}$ scheme, for $Y=\left(y_{1}, y_{2}, y_{3}\right)$ and $Z=\left(z_{1}, z_{2}, z_{3}\right)$ in $E^{3}$, we have

$$
\begin{aligned}
\left|\beta_{t, h}(Y)-\beta_{t, h}(Z)\right|_{\infty} \leq & \frac{11}{3} L_{b}|Y-Z|_{\infty} \\
& +\frac{h}{12}\left(\left|a\left(t, y_{3}\right) b\left(t-h, y_{2}\right)-a\left(t, z_{3}\right) b\left(t-h, z_{2}\right)\right|\right. \\
& \left.+\left|a\left(t-h, y_{2}\right) b\left(t, y_{3}\right)-a\left(t-h, z_{2}\right) b\left(t, z_{3}\right)\right|\right) .
\end{aligned}
$$

Let us bound the Lipschitz constant of a function of the type $F(Y)=a\left(\xi, y_{2}\right) b\left(\tau, y_{3}\right)$ for $0 \leq \tau, \xi \leq T$ :

$$
\begin{aligned}
|F(Y)-F(Z)| & =\left|a\left(\xi, y_{3}\right)\left(b\left(\tau, y_{2}\right)-b\left(\tau, z_{2}\right)\right)+\left(a\left(\xi, y_{3}\right)-a\left(\xi, z_{3}\right)\right) b\left(\tau, z_{2}\right)\right| \\
& \leq M_{a} L_{b}|Y-Z|_{\infty}+L_{a}|Y-Z|_{\infty}\left|b\left(\tau, z_{2}\right)\right| .
\end{aligned}
$$

With inequality (3.6), this yields, for $0 \leq \tau, \xi \leq T$ and $Y, Z$ in $E^{k}$,

$$
|F(Y)-F(Z)| \leq C_{3}|Y-Z|_{\infty}\left(1+|Z|_{\infty}\right) .
$$

As a result, we have

$$
\left|\beta_{t, h}(Y)-\beta_{t, h}(Z)\right|_{\infty} \leq C_{4}|Y-Z|_{\infty}\left(1+|Z|_{\infty}\right) .
$$

The same inequality holds for the $\mathrm{RL}_{4}$ scheme.

Finally, we consider $Y_{1}$ and $Y_{2}$ in $E^{k}$ and use the notation $\alpha_{i}=\alpha_{t, h}\left(Y_{i}\right)$ and $\beta_{i}=\beta_{t, h}\left(Y_{i}\right)$. Property (3.4) shows that $s_{t, h}\left(Y_{1}\right)-s_{t, h}\left(Y_{2}\right)=\left(z_{1}-z_{2}\right)(t+h)$, where $z_{i}$ is the solution to $z_{i}^{\prime}=\alpha_{i} z_{i}+\beta_{i}$ with $z_{i}(t)=Y_{i, k}$. On the one hand, with inequality (3.7), we have

$$
\left|z_{2}(\tau)\right| \leq C_{5}\left(1+\left|Y_{2}\right|_{\infty}\right)
$$

for $t \leq \tau \leq t+h$. On the other hand, on $[t, t+h]$, we have

$$
\begin{aligned}
\left|\left(z_{1}-z_{2}\right)^{\prime}\right| & \leq\left|\alpha_{1}\right|\left|z_{1}-z_{2}\right|+\left|\alpha_{1}-\alpha_{2}\right|\left|z_{2}\right|+\left|\beta_{1}-\beta_{2}\right| \\
& \leq M_{\alpha}\left|z_{1}-z_{2}\right|+L_{\alpha}\left|Y_{1}-Y_{2}\right|_{\infty} C_{5}\left(1+\left|Y_{2}\right|_{\infty}\right)+C_{4}\left|Y_{1}-Y_{2}\right|_{\infty}\left(1+\left|Y_{2}\right|_{\infty}\right) \\
& \leq M_{\alpha}\left|z_{1}-z_{2}\right|+C_{6}\left|Y_{1}-Y_{2}\right|_{\infty}\left(1+\left|Y_{2}\right|_{\infty}\right) .
\end{aligned}
$$

The initial condition yields

$$
\left|\left(z_{1}-z_{2}\right)(t)\right|=\left|Y_{1, k}-Y_{2, k}\right| \leq\left|Y_{1}-Y_{2}\right|_{\infty} .
$$

As a consequence, the Gronwall inequality (3.5) applied to these bounds shows that

$$
\begin{aligned}
\left|\left(z_{1}-z_{2}\right)(t+h)\right| & \leq \mathrm{e}^{M_{\alpha} h}\left(\left|Y_{1}-Y_{2}\right|_{\infty}+h C_{6}\left|Y_{1}-Y_{2}\right|_{\infty}\left(1+\left|Y_{2}\right|_{\infty}\right)\right) \\
& \leq \mathrm{e}^{M_{\alpha} h}\left|Y_{1}-Y_{2}\right|_{\infty}\left(1+C_{6} h\left(1+\left|Y_{2}\right|_{\infty}\right)\right) .
\end{aligned}
$$

This last inequality implies the stability condition (3.2), again by bounding the exponential with an affine function for $0 \leq h \leq T$. 


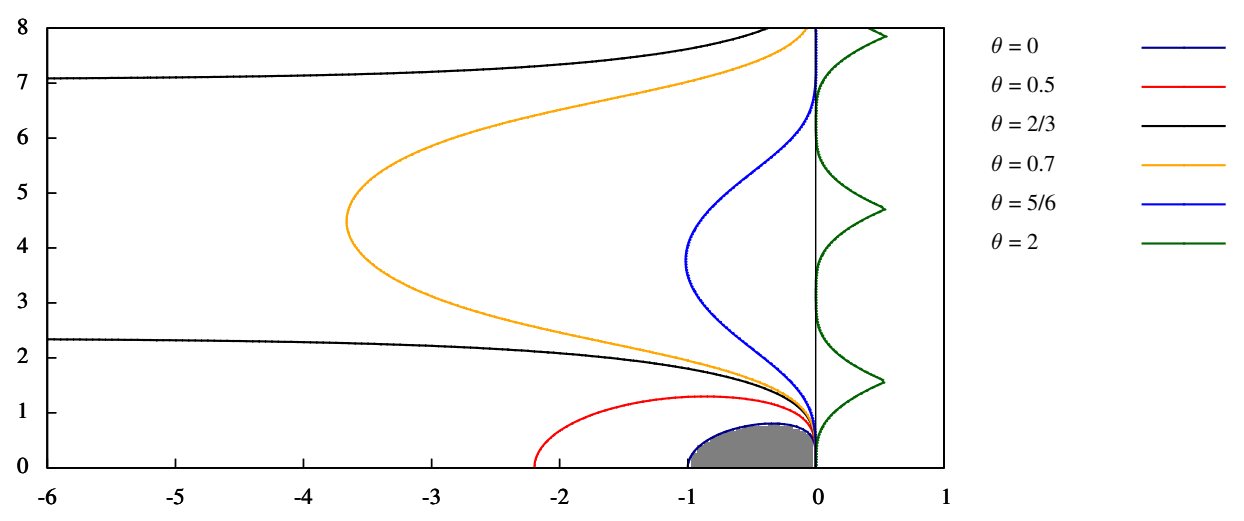

FIG. 4.1. Stability domain $D_{\theta}$ for the $R L_{2}$ scheme for various values of $\theta$. The stability domain for the particular case $\theta=0$ (no stabilization) is shown in gray, corresponding to the Adams-Bashforth scheme of order 2.

4. Dahlquist stability. For the general ideas and definitions concerning the Dahlquist stability we refer to [11]. The background for the Dahlquist stability of exponential integrators with a general varying stabilizer $a(t, y)$ has been developed in [6], following the ideas of Perego and Veneziani [25]. Equation (1.2) is considered with the Dahlquist test function $f(t, y)=\lambda y$, which is split into $f(t, y)=a(t, y) y+b(t, y)$ in order to match the framework of equation (1.3) with

$$
a(t, y)=\theta \lambda, \quad b(t, y)=\lambda(1-\theta) y
$$

For $\theta=1$, the methods are exact and thus $A$-stable. For $\theta \simeq 1$, the exact linear part of $f(t, y)$ in equation (1.2) is well approximated by $a(t, y)$. The stability domain depends on $\theta$, and it is denoted by $D_{\theta}$. Given a value of $\theta$, the region $D_{\theta}$ is defined by the modulus of a stability function with the same definition as for multistep methods; see, e.g., [11]. This stability function has been computed numerically, pointwise on a grid inside the complex plane $\mathbb{C}$ for each of the three $\mathrm{RL}_{k}$ schemes, $k=2,3,4$.

Order-2 Rush-Larsen. The stability domain for the $\mathrm{RL}_{2}$ scheme has been analyzed in [25]. The situation for this scheme is interesting, and we reproduced the results in Figure 4.1. We note the observations below.

- If $0 \leq \theta<2 / 3$, then the stability domain $D_{\theta}$ is bounded. Its size increases with $\theta$, starting from the stability domain without stabilization for $\theta=0$, which corresponds to the Adams-Bashforth scheme of order 2.

- If $\theta=2 / 3$, then the method is $A(0)$ stable: $\mathbb{R}^{-} \subset D_{\theta}$. The domain boundary is asymptotically parallel to the real axis so that the method is not $A(\alpha)$ stable.

- If $\theta>2 / 3$, then the stability domain is located around the $y$-axis: the method is $A(\alpha)$ stable. The angle $\alpha$ increases with $\theta$, and it goes to $\pi / 2$ as $\theta \rightarrow 1^{-}$.

Rush-Larsen methods of orders 3 and 4. The situation is different for the Rush-Larsen methods of orders 3 and 4. The stability domains $D_{\theta}$ are depicted in Figures 4.2 and 4.3 for various values of $\theta$ and for the orders 3 and 4, respectively. Except for the case $\theta=1$, the stability domain is always bounded: the scheme is not $A(0)$-stable. However, the stability domain for $\theta \simeq 1$ is much larger than without stabilization (corresponding to the AdamsBashforth schemes of orders 3 or 4 ). For the $\mathrm{RL}_{3}$ scheme, the stability domain when $\theta=0.85$ 


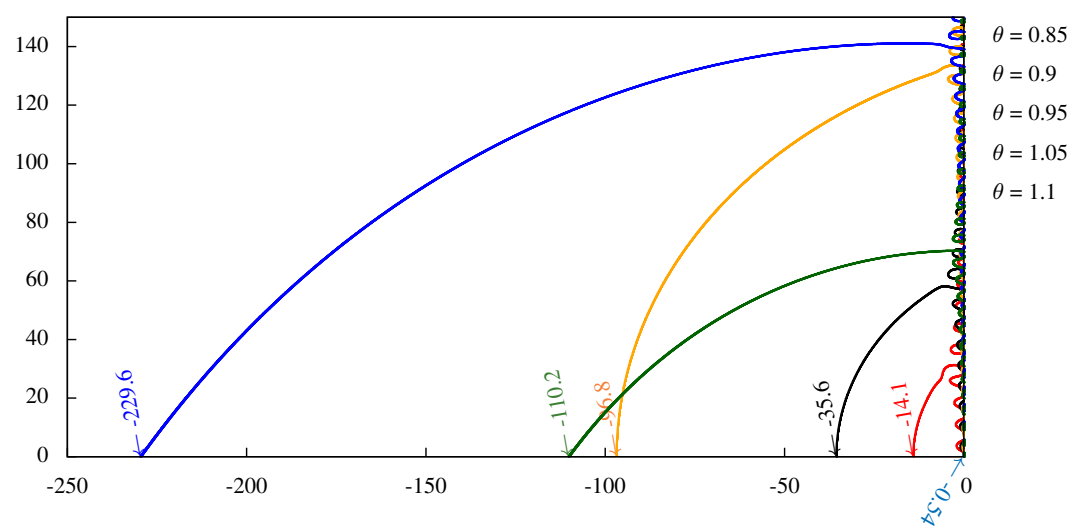

FIG. 4.2. Stability domain $D_{\theta}$ for the $R L_{3}$ scheme. In the particular case $\theta=0$ (no stabilization, corresponding to the Adams-Bashforth scheme of order 3 ), the stability domain crosses the $x$-axis at $x \simeq-0.54$ (dark blue arrow).

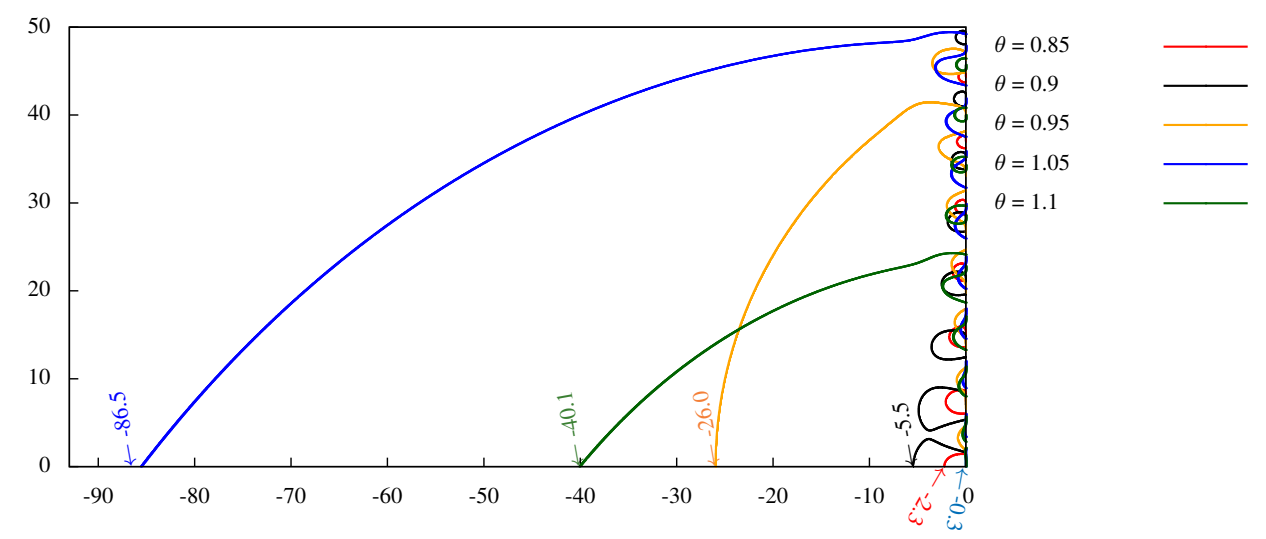

FIG. 4.3. Stability domain $D_{\theta}$ for the $R L_{4}$ scheme. In the particular case $\theta=0$ (no stabilization, corresponding to the Adams-Bashforth scheme of order 3 ), the stability domain crosses the $x$-axis at $x \simeq-0.3$ (dark blue arrow).

is 25 times wider on the left than $D_{\theta \mid \theta=0}$, and when $\theta=1.05$ it is 400 times wider. For the $\mathrm{RL}_{4}$ case, $D_{\theta \mid \theta=1.05}$ is almost 300 times wider on the left than $D_{\theta \mid \theta=0}$.

5. Numerical results. In this section we present numerical experiments that illustrate the performances of the $\mathrm{RL}_{k}$ methods. They will be compared to the exponential integrators of Adams type of order $k$ defined by equation (1.5), shortly denoted by $\mathrm{EAB}_{k}$. The $\mathrm{EAB}_{k}$ schemes have been numerically studied in [6] for the resolution of the membrane equation in electrophysiology and have been compared to several classical methods. In that context, they have been shown to be as stable as implicit methods with a much smaller cost. We present the same numerical tests here so as to extend the comparison to the schemes benchmarked in [6].

5.1. The membrane equation. The cellular action potential for cardiac cells is described in Figure 5.1. This phenomenon displays a stiff behavior characterized by the presence of heterogeneous time scales. The electrical activity of cardiac cells is modeled with an ODE 

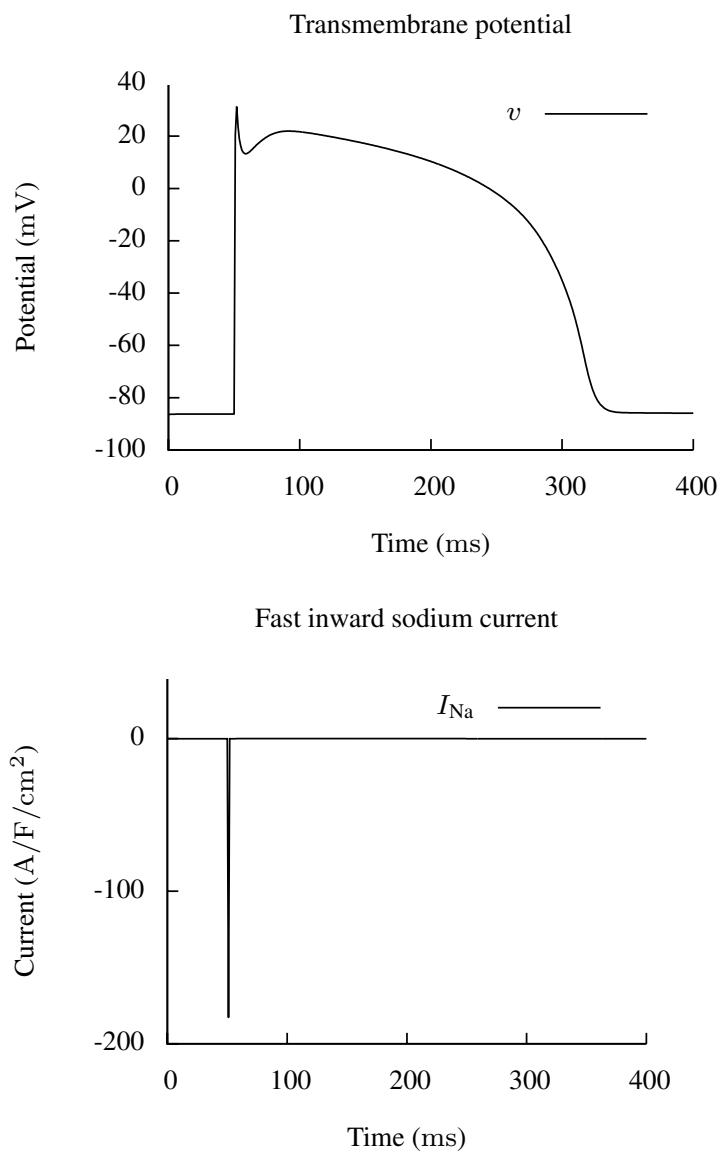

FIG. 5.1. TNNP model illustration. Top: cellular action potential: starting from a (negative) rest value, the transmembrane potential $v(t)$ has a stiff depolarization followed by a plateau and a repolarization to the rest value. Bottom: depolarization is induced by an ionic sodium current $I_{N a}$ with obvious large stiffness.

system called membrane equation. It has the form

$$
\frac{\mathrm{d} v}{\mathrm{~d} t}=-I_{i o n}(v, w, c)+I_{s t}(t), \quad \frac{\mathrm{d} w_{i}}{\mathrm{~d} t}=\frac{w_{\infty, i}(v)-w_{i}}{\tau_{i}(v)}, \quad \frac{\mathrm{d} c}{\mathrm{~d} t}=g(v, w, c)
$$

where $w=\left(w_{1}, \ldots, w_{p}\right) \in \mathbb{R}^{p}$ is a vector of gating variables, $c \in \mathbb{R}^{q}$ is a vector of ionic concentrations, and $v \in \mathbb{R}$ is the transmembrane potential; we refer to [1, 19, 22, 29] for details. The gating variables describe the opening state (between 0 and 1) of various protein structures on the cell membrane, which control ionic transfers between the intra and extra-cellular media. Each gating variable $w_{i}$ evolves towards the state $w_{\infty, i}(v)$ at a rate $\tau_{i}(v)$. Specific ionic currents (sodium, potassium, ...) across the cellular membrane are computed with the help of the variables $v, w$, and $c$. The sum of these currents defines the total ionic current $I_{i o n}(v, w, c)$ across the membrane. The function $I_{s t}(t)$ is a source term; it represents a stimulation current. The membrane equation corresponds to the ODE system in the monodomain model (1.1) with $\zeta=(w, c)$.

We will consider two such models: the BR model [1] and the TNNP model [29]. The BR model [1] describes the membrane action potential of mammalian ventricular myocardial cells. It involves 6 gating variables ( $p=6$, denoted by $\left.m, h, j, d, f, x_{i}\right)$ and one ionic concentration $(q=1)$ : the intra-cellular calcium $\left[\mathrm{Ca}_{i}\right]$. The Nernst potential $E_{\mathrm{Ca}}$ for the calcium ions then 


\section{ETNA}

Kent State University and

Johann Radon Institute (RICAM)

is time-dependent and a (slow inward) calcium current $I_{s}$ is modeled as $I_{s}=g_{s} d f\left(v-E_{\mathrm{Ca}}\right)$ depending on the gating variables $d, f$, the transmembrane potential $v$, and a constant $g_{s}$. A fast inward sodium current $I_{\mathrm{Na}}$ (depicted in Figure 5.1) that depends on the three gating variables $m, h, j$ and on $v$ is similarly described. Two outward currents are modeled: $I_{x_{1}}$, which depends on $x_{1}$ and $v$ and $I_{K_{1}}$, which only depends on $v$. The total ionic current in (5.1) is the sum of these four currents $I_{\mathrm{Na}}+I_{s}+I_{K_{1}}+I_{x_{1}}=-I_{\text {ion }}(w, c, v)$.

The TNNP model is specifically designed for human ventricular myocytes. It is more sophisticated than the BR model but conserves the same general structure. It involves 12 gating variables and 4 ionic concentrations $(p=12$ and $q=4)$. The total ionic current $I_{\text {ion }}$ is the sum of 15 specific ionic currents.

The membrane equation (5.1) can be reformulated in the form of (1.3) with

$$
a(t, y)=\left[\begin{array}{ccc}
-1 / \tau(v) & 0 & 0 \\
0 & 0 & 0 \\
0 & 0 & 0
\end{array}\right], \quad b(t, y)=\left[\begin{array}{c}
w_{\infty}(v) / \tau(v) \\
g(y) \\
-I_{\text {ion }}(y)+I_{s t}(t)
\end{array}\right]
$$

for $y=(w, c, v) \in \mathbb{R}^{N}(N=p+q+1)$, and where $-1 / \tau(v)$ is the $p \times p$ diagonal matrix with diagonal entries $\left(-1 / \tau_{i}(v)\right)_{i=1, \ldots, p}$. The resulting matrix $a(t, y)$ is diagonal.

5.2. Convergence. No analytical solution is available for the chosen application. A reference solution $y_{\text {ref }}$ for a reference time step $h_{\text {ref }}$ is computed with the Runge-Kutta scheme of order 4 to analyze the convergence properties of the $\mathrm{RL}_{k}$ schemes. Numerical solutions $y$ are compared to $y_{\text {ref }}$ for the coarsest time steps $h=2^{m} h_{\text {ref }}$.

A numerical solution $y$ consists in successive values $y_{n}$ at the time instants $t_{n}=n h$. On every interval $\left(t_{3 n}, t_{3 n+3}\right)$, the polynomial $\bar{y}$ of degree at most 3 is constructed so that $\bar{y}\left(t_{3 n+i}\right)=y_{3 n+i}, i=0, \ldots, 3$. On $(0, T), \bar{y}$ is continuous and a piecewise polynomial of degree 3 , and its values at the reference time instants $n h_{\text {ref }}$ are computed. This provides a projection $P(y)$ of the numerical solution $y$ onto the reference grid. Then, $P(y)$ can be compared with the reference solution $y_{\text {ref. }}$. The numerical error is defined by

$$
e(h)=\frac{\max \left|v_{\text {ref }}-P(v)\right|}{\max \left|v_{\text {ref }}\right|},
$$

where the potential $v$ is the last and stiffest component of $y$ in equation (5.1). The convergence graphs for the BR model are displayed in Figure 5.2. Each scheme shows the expected asymptotic behavior of Proposition 3.1: $e(h)=O\left(h^{k}\right)$ as $h \rightarrow 0$.

5.3. Stability. Spiteri et al. in [28] have evaluated the stiffness of the BR and TNNP models along one cellular action potential (as depicted in Figure 5.1). The largest negative real part of the eigenvalues of the Jacobian matrix during the action potential is -1170 and -82 for the TNNP and BR models, respectively. The TNNP model thus is 15 times stiffer than the BR model $(15 \simeq 1170 / 82)$.

Robustness to stiffness for the $\mathrm{RL}_{k}$ schemes is evaluated by comparing the critical time steps for these two models. The critical time step $\Delta t_{0}$ is defined as the largest time step such that the numerical simulations run without overflow for $h<\Delta t_{0}$. The results are presented in Table 5.1.

An excellent robustness to stiffness can be observed. The $\mathrm{RL}_{k}$ schemes are not $A(\alpha)$ stable, and the critical time step is expected to be divided by 15 in case of an increase of stiffness of magnitude 15. It is here divided by $2.7,2.0$, and 1.3 for $k=2,3$, and 4, respectively. A comparison with the $\mathrm{EAB}_{k}$ schemes shows that the two schemes have similar robustness to stiffness. Loss of stability is induced by the non-stabilized part, whose eigenvalues are less modified by the change of the model. 


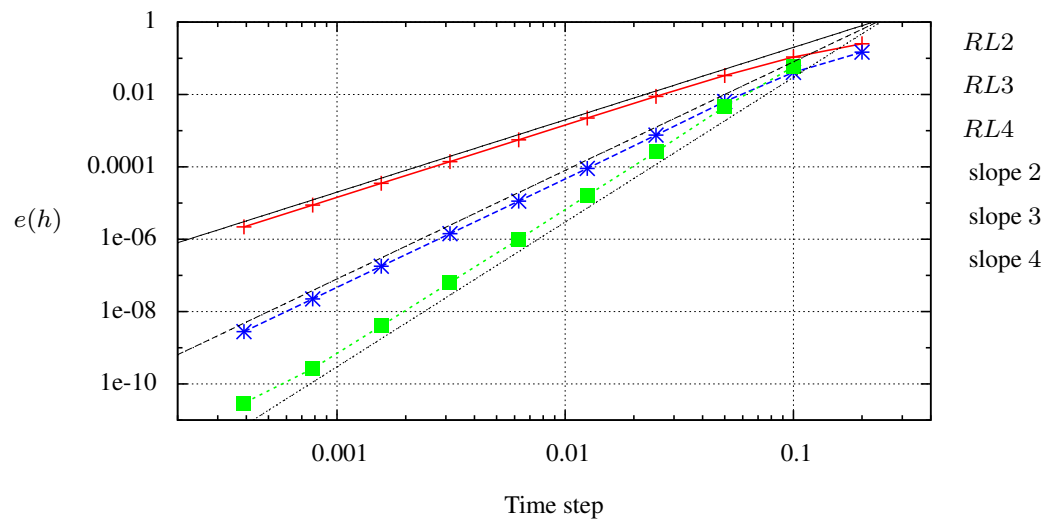

FIG. 5.2. Relative error e $(h)$ (definition (5.2)) as a function of the time step $h$ for the $R L_{k}$ schemes, for $k=2$ to 4 and in Log/Log scale.

TABLE 5.1

Critical time steps $\Delta t_{0}$ for the $R L_{k}$ and $E A B_{k}$ schemes.

\begin{tabular}{c|c|c|c||c|c|c} 
method & $\mathrm{RL}_{2}$ & $\mathrm{RL}_{3}$ & $\mathrm{RL}_{4}$ & $\mathrm{EAB}_{2}$ & $\mathrm{EAB}_{3}$ & $\mathrm{EAB}_{4}$ \\
\hline BR & 0.323 & 0.200 & 0.149 & 0.424 & 0.203 & 0.123 \\
TNNP & 0.120 & 0.148 & 0.111 & 0.233 & 0.108 & $7.5610^{-2}$
\end{tabular}

5.4. Accuracy. The $\mathrm{RL}_{k}$ schemes are compared to the $\mathrm{EAB}_{k}$ schemes in terms of accuracy. This is done using the relative error $e(h)$ in equation (5.2) for the BR and TNNP models (we recall than the TNNP model is stiffer by a factor of 15). The results are collected in Tables 5.2 and 5.3.

TABLE 5.2

Relative error $(h)$ (equation (5.2)) for the BR model.

\begin{tabular}{c|c|c|c||c|c|c}
$h$ & $\mathrm{RL}_{2}$ & $\mathrm{RL}_{3}$ & $\mathrm{RL}_{4}$ & $\mathrm{EAB}_{2}$ & $\mathrm{EAB}_{3}$ & $\mathrm{EAB}_{4}$ \\
\hline 0.2 & 0.251 & 0.147 & - & 0.284 & 0.516 & - \\
0.1 & 0.107 & $4.0710^{-2}$ & $5.8610^{-2}$ & $9.2610^{-2}$ & $9.1710^{-2}$ & 0.119 \\
0.05 & $3.3510^{-2}$ & $6.3410^{-3}$ & $4.5810^{-3}$ & $2.3110^{-2}$ & $1.0910^{-2}$ & $8.9610^{-3}$ \\
0.025 & $8.8810^{-3}$ & $7.5710^{-4}$ & $2.6110^{-4}$ & $5.3910^{-3}$ & $1.1710^{-3}$ & $4.3310^{-4}$
\end{tabular}

TABLE 5.3

Relative error e $(h)$ (equation (5.2)) for the TNNP model.

\begin{tabular}{c|c|c|c||c|c|c}
$h$ & $\mathrm{RL}_{2}$ & $\mathrm{RL}_{3}$ & $\mathrm{RL}_{4}$ & $\mathrm{EAB}_{2}$ & $\mathrm{EAB}_{3}$ & $\mathrm{EAB}_{4}$ \\
\hline 0.1 & 0.177 & 0.305 & 0.421 & 0.351 & 0.530 & - \\
0.05 & $7.3910^{-2}$ & $4.5410^{-2}$ & $4.6110^{-2}$ & $9.0110^{-2}$ & $5.5910^{-2}$ & $8.9310^{-2}$ \\
0.025 & $2.2110^{-2}$ & $6.5310^{-3}$ & $5.9610^{-3}$ & $2.1410^{-2}$ & $7.3410^{-3}$ & $8.3410^{-3}$ \\
0.0125 & $5.7510^{-3}$ & $8.0510^{-4}$ & $3.2110^{-4}$ & $5.1110^{-3}$ & $7.6210^{-4}$ & $3.7010^{-4}$
\end{tabular}

For the $\mathrm{RL}_{2}$ and the $\mathrm{EAB}_{2}$ schemes, the accuracies are very close, with the $\mathrm{EAB}_{2}$ scheme being slightly more accurate for the BR model. For the orders 3 and 4 , the $\mathrm{RL}_{k}$ schemes are 
more accurate at large time steps. For smaller time steps, the accuracies are almost the same. The $\mathrm{RL}_{k}$ and $\mathrm{EAB}_{k}$ have the same accuracy in the asymptotic convergence region.

6. Conclusion. In this paper, we have introduced two new ODE solvers called RushLarsen schemes of orders 3 and 4 . They are explicit multistep exponential integrators. Their definition is simple inducing an easy implementation. We have presented the analysis of convergence and of stability under perturbation for these two schemes. We have also analyzed their Dahlquist stability: they are not $A(0)$ stable but exhibit very large stability domains for sufficiently accurate stabilization. The numerical behavior of the schemes is analyzed for a complex and realistic stiff application. The $\mathrm{RL}_{k}$ schemes are as stable as exponential integrators of Adams type, allowing simulations at large time steps. For the presented example, the $\mathrm{RL}_{k}$ schemes are more accurate for $k=3$ and 4 than the exponential integrators of Adams type when considering larger time steps. They are also shown to be robust to stiffness in terms of both stability and accuracy.

Acknowledgments. This study received financial support from the French government as part of the "Investissement d'Avenir" program managed by the "Agence Nationale de la Recherche" (ANR), grant reference ANR-10-IAHU-04. It also received fundings of the ANR project HR-CEM, grant reference 13-MONU-0004-04.

\section{REFERENCES}

[1] G. BEELER AND H. REUTER, Reconstruction of the action potential of ventricular myocardial fibres, J. Physiol., 268 (1977), pp. 177-210.

[2] M. CHU, An automatic multistep method for solving stiff initial value problems, J. Comput. Appl. Math., 9 (1983), pp. 229-238.

[3] J. Clements, J. Nenonen, P. LI, And B. HorÁCeK, Activation dynamics in anisotropic cardiac tissue via decoupling, Ann. Biomed. Eng., 32 (2004), pp. 984-990.

[4] P. Colli-Franzone, L. PaVArino, And B. TACCARdi, Monodomain simulations of excitation and recovery in cardiac blocks with intramural heterogeneity, in Functional Imaging and Modeling of the Heart, A. F. Frangi, P. I. Radeva, A. Santos, M. Hernandez, eds., vol. 3504 of Theoretical Computer Science and General Issues, Springer, Berlin, 2005, pp. 267-277.

[5] - Simulating patterns of excitation, repolarization and action potential duration with cardiac bidomain and monodomain models, Math. Biosci, 197 (2005), pp. 35-66.

[6] Y. Coudiére, C. Douanla-Lontsi, And C. Pierre, Exponential Adams Bashforth integrators for stiff ODEs, application to cardiac electrophysiology, Math. Comput. Simulation, 153 (2018), pp. 15-34

[7] S. Cox And P. Matthews, Exponential time differencing for stiff systems, J. Comput. Phys., 176 (2002), pp. 430-455.

[8] S. DRAGOMIR, Some Gronwall Type Inequalities and Applications, Nova Science Publishers, Hauppauge, 2003.

[9] C. González, A. Ostermann, And M. Thalhammer, A second-order Magnus-type integrator for nonautonomous parabolic problems, J. Comput. Appl. Math., 189 (2006), pp. 142-156.

[10] E. Hairer, S. NøRSetT, AND G. WANner, Solving Ordinary Differential Equations I, Springer, Berlin, 1993.

[11] E. HaIRer And G. WANnER, Solving Ordinary Differential Equations II, Springer, Berlin, 1996.

[12] M. HochbrUCK, A short course on exponential integrators, in Matrix Functions and Matrix Equations, Z. Bai, W. Gao, and Y. Su, eds., vol. 19 of Contemp. Appl. Math., Higher Ed. Press, Beijing, 2015, pp. 28-49.

[13] M. HochBRUCK AND C. LUBICH, On Magnus integrators for time-dependent Schrödinger equations, SIAM J. Numer. Anal., 41 (2003), pp. 945-963.

[14] M. HochbrUCK, C. LUBICH, AND H. SELHOFER, Exponential integrators for large systems of differential equations, SIAM J. Sci. Comput., 19 (1998), pp. 1552-1574.

[15] M. Hochbruck AND A. Ostermann, Explicit exponential Runge-Kutta methods for semilinear parabolic problems, SIAM J. Numer. Anal., 43 (2005), pp. 1069-1090.

[16] - Exponential integrators, Acta Numer., 19 (2010), pp. 209-286.

[17] - Exponential multistep methods of Adams-type, BIT, 51 (2011), pp. 889-908.

[18] M. Hochbruck, A. Ostermann, AND J. SCHWeitzer, Exponential Rosenbrock-type methods, SIAM J. Numer. Anal., 47 (2009), pp. 786-803. 
[19] A. Hodgkin And A. Huxley, A quantitative description of membrane current and its application to conduction and excitation in nerve, J. Physiol., 117 (1952), pp. 500-544.

[20] D. LEE AND S. PREISER, A class of non linear multistep A-stable numerical methods for solving stiff differential equations, Comput. Math Appl., 4 (1978), pp. 43-51.

[21] V. LuAn And A. Ostermann, Explicit exponential Runge-Kutta methods of high order for parabolic problems, J. Comput. Appl. Math., 256 (2014), pp. 168-179.

[22] C. H. LUO AND Y. RUDY, A dynamic model of the cardiac ventricular action potential, Circ. Res., 74 (1994), pp. 1071-1096.

[23] B. MincheV AND W. WRIGHT, A review of exponential integrators for first order semi-linear problems, Preprint Numerics 2/2005, Norges Teknisk-Naturvitenskapelige Universitet, Trondheim, 2005.

[24] S. NøRSETt, An A-stable modification of the Adams-Bashforth methods, in Conference on the Numerical Solution of Differential Equations 1969, J. L. Morris, ed., Springer, Berlin, 1969, pp. 214-219.

[25] M. PEREgo And A. Veneziani, An efficient generalization of the Rush-Larsen method for solving electrophysiology membrane equations, Electron. Trans. Numer. Anal., 35 (2009), pp. 234-256. http://etna.ricam.oeaw.ac.at/vol.35.2009/pp234-256.dir/pp234-256.pdf

[26] G. RAINWATER AND M. TOKMAN, A new class of split exponential propagation iterative methods of RungeKutta type (sEPIRK) for semilinear systems of ODEs, J. Comput. Phys., 269 (2014), pp. 40-60.

[27] S. RUSH AND H. LARSEN, A practical algorithm for solving dynamic membrane equations, IEEE Trans. Biomed. Eng., 25 (1978), pp. 389-392.

[28] R. J. SPIteri AND R. C. DEAn, Stiffness analysis of cardiac electrophysiological models, Ann. Biomed. Eng., 38 (2010), pp. 3592-3604.

[29] K. ten Tusscher, D. Noble, P. Noble, And A. Panfilov, A model for human ventricular tissue, Am. J. Physiol. Heart Circ. Physiol., 286 (2004), pp. H1573-H1589.

[30] M. TOKMAN, J. LofFeld, AND P. TRANQUILLI, New adaptive exponential propagation iterative methods of Runge-Kutta type, SIAM J. Sci. Comput., 34 (2012), pp. A2650-A2669.

[31] P. TRAnQuilli And A. SAndu, Rosenbrock-Krylov methods for large systems of differential equations, SIAM J. Sci. Comput., 36 (2014), pp. A1313-A1338. 